\title{
EVOLVED CONSCIOUSNESS
}

\author{
Richard A. Mould* \\ Department of Physics and Astronomy, State University of New York, \\ Stony Brook, N.Y. 11794-3800, USA. \\ Received December 12, 2010; accepted December 18, 2010
}

\begin{abstract}
The purpose of consciousness in primitive creatures is found to be the repression or enhancement of completing pathways of evolution. In advanced creatures such as humans the purpose of consciousness has become the repression or enhancement of competing neurological pathways. The 'causal' influence of consciousness is the same in both cases. A previous paper describes the introduction of pain and fear consciousness in a primitive fish, and the intermediate introduction of the fish's visual experience. The manner of introduction of these conscious experiences is essential to their matching physical behavior in the right way - so subjective experiences emerge in parallel with the behaviors to which they are appropriately attached. When the same cause-and-effect principles are applied to pleasure and desire consciousness, familiar human patterns emerge. Novel behaviors such as the neuromotor control of a prosthetic device are also explained in this way.
\end{abstract}

Key words: Consciousness; Desire; Pain; Physics; Repression

\section{BACKGROUND}

It is proposed in a previous paper that consciousness is introduced into a species by a genetic mutation in such a way that consciousness and physiology evolve in parallel with one another. That is, subjective experiences will follow and reflect evolving physical behaviors (Mould, 2009a). It is assumed that organisms first evolve without the aid of consciousness, but that at some point a mutation introduces consciousness into a stimulusresponse process that either supports or represses that process. The survival chances of the individual are thereby enhanced or diminished, and this in turn supports the survival or extinction of the species.

These mechanics insure that a species that emerges from a long evolution will enjoy a parallel and harmonious relationship between its physical and its psychic life.

In a fanciful example we imagine a fish that evolves without consciousness. It is an automaton - a robofish. Through a process of evolutionary weeding out it has come to do all the things it needs to do to survive. It swims about, eats and digests, reproduces, and avoids life threatening predators; and in our particular example, it has learned to avoid jellyfish tentacles. We then imagine that robofish undergoes a mutation that introduces pain consciousness. Pain consciousness has the property that it always represses any behavior to which it becomes associated, as shown in Fig. 1. The fish has already learned to withdraw from contact with jellyfish tentacles with a suitable degree of probability, but there remains some chance that it will continue contact. The fish may not have learned its lesson very well, so pain consciousness adds a further incentive that can go in either direction. If pain becomes associated with the 'withdraw' behavior in Fig. 1, then continued contact with the tentacles will be encouraged and this will lead to the demise of the fish. But if the introduced pain is associated with 'continued contact' with the tentacles in Fig. 1, then that behavior will be repressed and the fish will better survive. This mechanism insures that pain will be appropriately associated with tentacle contact in the surviving species - the psychological will be harmonious with the physical.

This intervention was not necessary for survival. Robofish managed to get along without it. But somewhere along the line consciousness was nonetheless introduced. Perhaps it was more efficient. Maybe it provided a faster way of doing things than the old autonomic way. We don't know enough about consciousness at this point to say 'why' it came to characterize a separate 
branch of evolution, but we know that it did. It gave us a psychic life that is parallel to and harmonious with our physical development.

Figure 1 also shows pain consciousness as spanning the entire behavior rather than applied to the stimulus or response alone. This is a continuous process in which stimulus and response are not always clearly differentiated; so consciousness is associated with the entire behavior, even if its parts are slightly separand in time.

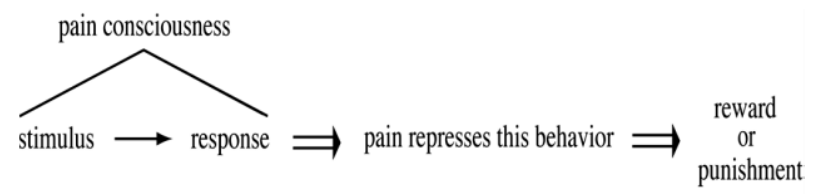

Figure 1. Pain repression of a behavior leads to reward or punishment

Pain and pleasure are called anchor experiences in another previous paper because they both have a causative influence on behavior (Mould, 2009b). Just as pain represses abehavior with which it is associated, pleasure enhances a behavior. Both are emotions that have a direct motivating influence on the body of the subject. On the other hand, vision is a phenomenon that appears outside the body and does not (by itself) motivate anything. If the fish only sees the jellyfish tentacles in the absence of any other experience, past or present, it will have no reason to be alarmed. The dashed line associated with vision in Fig. 2 means that it is a subjective experience that appears outside the body of the fish and is not an anchor experience. Vision does not by itself repress or enhance behavior.

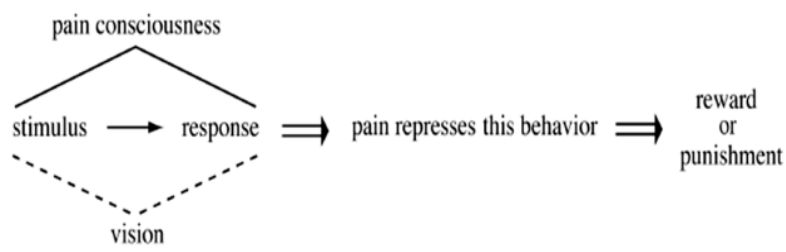

Figure 2. Pain repression accompanied with vision.

Then what is the point of vision? Why should vision be added to the fish's experience if it cannot (by itself) contribution causally to an outcome? It may have been added for one of the reasons given in (Mould, 2009b) that have nothing to do with this particular behavior; but once established, it might lead to an important further development. The fish might see the nearby tentacles before contact, so it might see disaster coming over its horizon. If the fish regularly sees the jellyfish tentacles before contact, than we imagine that a further mutation will introduce another anchor experience fear that also can motivate a response. When favorably introduced, fear consciousness will repress the behavior 'before' contact (Fig. 3). Like pain it will always repress a beha- vior; but unlike pain, it is not likely to come into existence on its own. The creature will not have a fear experience without first experiencing something to be afraid of, and this suggests that pain and vision will precede fear.

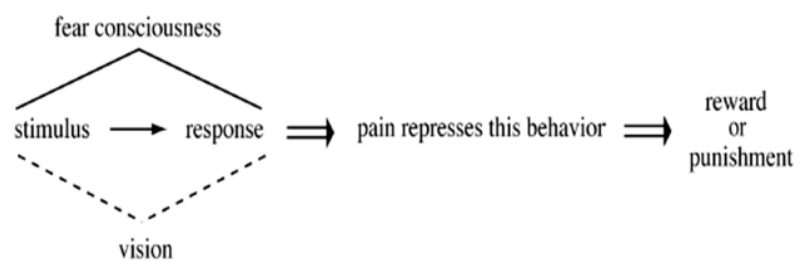

Figure 3. Fear repression accompanied with vision.

These mutations: Pain that represses behavior, fear that represses behavior, and vision that is a causal, will produce a species of fish whose subjective states will support its physiological behaviors. When it encounters the tentacles of a jellyfish it will experience pain and swim away; and when it sees approaching jellyfish tentacles it will experience fear and swim away. We may not understand how or why these experiences are introduced in this particular way, but when that happens these survival patterns will emerge.

\section{AS EFFECTS}

Pain and fear have so far functioned as causes. They have 'caused' the repression of the behaviors with which they are associated. But nothing in nature is a cause alone or an effect alone. Everything is simultaneously both. Furthermore, it is not clear from our description how consciousness becomes attached to one stimulus and not another. We have only said that it becomes attached to the stimulus that we happen to be discussing, as though a separate mutation is required for each separate behavior.

To complete the picture we must require that each behavior somehow causes the particular form of consciousness with which it is associated. A biological mutation is an extraordinary event that empowers a certain configuration of neurons and the associated body and behavioral parts to 'cause' consciousness, where the relevant parts are already present in the automaton. The robofish already has a nociceptive nervous system that initiates a withdrawal when its body is harmed in some way. It is the behavior involving this system that the mutation endows with a conscious experience of pain that will appear anytime the behavior appears, and will ultimately support a healthy withdrawal. The mutation therefore introduces a causal 'loop' in which pain consciousness is caused by an assault on a nervous systems that is already present in the primitive fish, and pain in turn represses the behavior that gives rise to it.

The same may be said of fear consciousness, although there is a very different neurological configuration in 
this case. The physiology of fear involves the primitive amygdale of the fish that is stimulated in one way or another by all of its senses. Fear is a therefore a very different emotion than pain and requires a separate mutation; although the two are functionally related in such a way that fear can be said to derive from pain.

\section{VISION}

Like pain and fear, vision is an experience that covers an entire behavior as shown in Figs. 2 and 3. It is not simply a response to a stimulus of some kind on the retina, or a specific configuration of neurons that fire in the visual cortex. Visual experience extends all the way to the level of behavior as is demonstrated in the classic experiments with visual inversion.

In visual inversion, a subject wears an optical apparatus that inverts the retinal image so that the world appears upside-down and/or left-right inverted. At first the subject is disoriented, but after a time he learns to see things correctly. This recovery is due to the amazing plasticity of the brain. The brain is able to rewire itself to 'right the image' so the subject can use vision to maneuver about the world in a causally effective way. That is, his vision again performs in the same space as his arms and legs. This conversion to normality does not occur 'at once' throughout the visual field, but applies first to those parts of the visual field that involve some activity. Between the onset of the experiment and the time of complete accommodation (as much as two or three weeks later), the subject will experience a fragmented visual field where some parts have been righted while other parts remain inverted. If the subject is using tools, it is the tools that first become erect while the background remains inverted. After 18 days of wearing reversing glasses a subject standing on a curb is reported to correctly see cars moving to his right, but he continues to see license plates numbers as mirror images (Kohler, 1951; O'Regan, \& Noë, 2001). Clearly vision is not just a function of where and how the retina is stimulated, but it also has an apparent relationship to attention and behavior. This includes how a subject becomes consistently involved in a whole body experience such as seeing a car go to the right at the same time as hearing it go to the right; or lifting an object up and seeing it go up. More generally Noë says, "consciousness is a world-involving dynamic process" (Noë, 2009).

We have talked about the introduction of a "vision mutation" as though it must have happened all at once. That is probably not correct. The biological introduction of vision was probably fragmented as it is in the case of inverted glasses, where the first additions to vision were those parts of the environment that were of greatest working interest to the species. Furthermore, this process may have happened over a span of time that included more than one species - not just a fish. It may also have happened in concert with other non-anchor sensations like tactual and auditory input, as well as in conjunction with anchor experiences like smell and taste that are heightened by hunger. So the first object appearing in a conscious representation of three dimensional space may have been the victim of feasting behavior; and that that flora's or fauna's 'objectification' (in consciousness) may have taken place over many mutations covering many species, and coincident with the gradual phasing in of a number of different kinds of contributing experiences like vision. Nonetheless, I will continue to cut through these many machinations by imagining that single mutations were responsible for the introduction of entire modes of experience in a single species. This idealization simplifies and I believe clarifies the role of different kinds of mutations.

\section{PLEASURE AND DESIRE}

Pleasure is another anchor experience like pain, except that it enhances a behavior instead of repressing it (Mould, 2009a). It engages very different physiological components than does pain, such as endorphins attaching to opiate receptors; so the introduction of pleasure consciousness must have resulted from a separate mutation. This might have occurred before or after the introduction of pain consciousness. Since opiate receptors appear in all vertebrates it might have happened before; but for the purpose of discussion we will assume that pleasure consciousness happened to robofish at about the same time as pain consciousness.

Imagine that robofish is attracted to food, which must certainly be the case if it survives at all. This attraction originally takes the form of an automatonic stimulus and response of the kind pictured in Fig. 4. The introduction of pleasure consciousness has the effect of enhancing the associated behavior that is similar to the way that pain represses behavior; so the diagram in Fig. 4 for pleasure is similar to Fig. 1 for pain. If pleasure consciousness is applied to a withdraw behavior from the food, then the fish will be selected against. If it is applied to eating the food, then the fish will be favored by evolution. These mechancs will support the survival of a species of fish that derives pleasure from eating food.

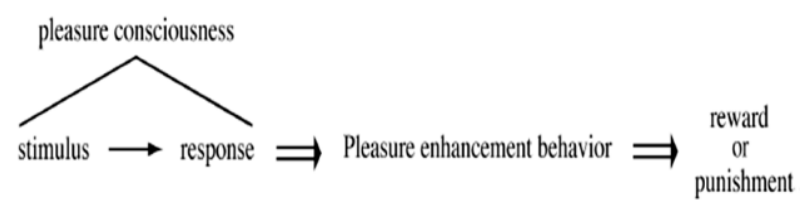

Figure 4. Pleasure enhancement fo a behavior leads to reward or punishement.

If the fish also has the ability to consciously 'see' food, it can anticipate pleasure in the same way that it antic- 
ipates pain when it sees jellyfish tentacles. The anticipation of pain we have seen to be the anchor experience 'fear'. In a similar way we introduce the anchor experience desire that anticipates pleasure. This is shown in Fig 5 analogous to Fig. 3. When the fish sees food it experiences desire in anticipation of a pleasurable feast, and that experience causally enhances its approach to the food.

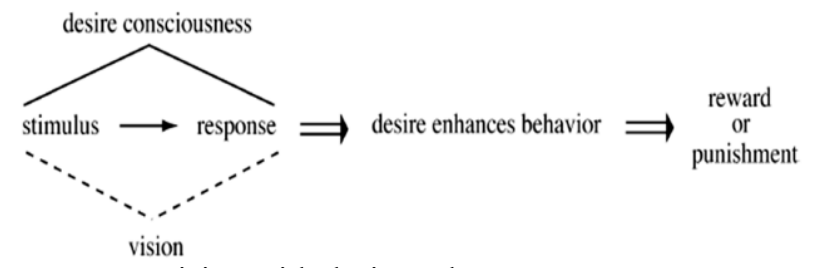

Figure 5. Vision with desire enhancement.

When a human desires the keys to his car he reaches for them following the pattern in Fig. 5. Researchers prefer to use the word 'intension' rather than desire, probably because it seems less emotionally charged. They call 'reaching for the keys' an intension-driven behavior. Desire and intention are of course the same thing with different emotional intensities. They are both anchor experiences that enhance the behavior to which they are attached, and they are both emotions as are all anchor experiences. I prefer to use the word desire because it does not bury the emotional aspect of this experience.

\section{A NEW ROLE}

Anchor experiences were introduced in the fish to play a role in the survival of the species. We humans have not escaped evolution, but these conscious experiences have taken on a different role in humans. They enable the human individual to learn new behaviors without having to undergo new biological mutations.

Imagine that a human has the desire to learn a new behavior. He desires to twirl a baton with his left hand and he has never done that before. He begins by picking up the baton with his left hand, a behavior that is accompanies by a desire to do so (as in Fig. 5). Desire causally supports rather than represses that behavior. If his next move fails to get the desired result the immediate visual and tactual feedback will produce something akin to 'a fear of failure' that will repress the associated behavior. Again, I prefer to say 'fear of failure' rather than something more emotionally neutral like 'disappointment'. It makes a stronger connection to the physiology of fear that has already been established. In any case the repressed behavior includes all of the neurological activity that completes the loop from the sensory organs to the motor cortex, and from there to the fingers of the left hand.

The human tries again. He again picks up the baton with his left hand, a behavior that is again accompanied by a desire to twirl the baton. The previously tried neurological route has been repressed, so the brain tries another path going from the motor cortex to the fingers. If it succeeds this time, pleasure will support the new behavior and will reinforce the neurological pathway chosen on this occasion. The first pathway chosen was repressed by a 'fear of failure', but the second pathway was enhanced by the 'pleasure of success'. At the level of humans, the causal influence of consciousness has more to do with the repression or enhancement of neurological pathways than it does the pathways of evolution. This is made possible by the plasticity of the human nervous system. As in the case of visual inversion, learning flexibility in this modern sense appears to be the great advantage of consciousness; although again, we cannot say that a robot cannot evolve with these same capabilities. However, we do know that our species has not taken the robotic route. For better or for worse, our line of evolution has made use of consciousness.

\section{NEUROMOTOR PROSTHESES}

When a human reaches for the keys he has the impression that it is his desire for the keys that empowers the response of his arms and fingers. That is correct. It even works when the neural pathways from his brain to his limbs have been artificially replaced.

The conscious desire to reach for something is caused by neuronal activity in the brain that is detectable on that part of the motor cortex that governs the arm, hand and fingers. This desire enhances the neural pathways that respond successfully to these commands, thereby completing a causal loop that we call a learning experience. If the subject does not have an arm, hand or fingers, the initiating activity in the motor cortex can be used to activate a prosthesis that performs the desired task - retrieving the keys to the car. Visual feedback then informs the subject of his success, and that completes the learning loop (Hoshberg, et al., 2006).

However sophisticated the electronics that connect the neuralmotor activity with the prosthesis, the result is likely to be crude compared to the smooth motion that is achieved by intact limbs. But brain plasticity allows alternative neural pathways to be found in the motor cortex as the subject visually monitors his failure or success. After some practice, fairly smooth command of the prosthesis is achieved in this way.

If the subject is asked how he does it, he will respond in the same way that you or I would respond to the same question. We say: "I desire (or intend) to reach for the keys and it just happens". This leaves out all of the neurological detail, but the answer is essentially correct. Desire is a causal element in this process. It enhances the behavior that carries out the desired response.

There are those who would use the word "will" instead of desire or intent, and who go further to call it "free 
will". The term 'will' is all right but it is not free. There is nothing in the post-big-bang universe that is free of contingencies, or circumstances or initial conditions. Human 'will' does not exist independent of neurological configurations that are themselves prompted by external conditions. Nonetheless, it is itself causal in its interaction with physiology.

\section{THE PURPOSE OF CONSCIOUSNESS}

Consciousness was originally introduced as a selection mechanism in the survival struggle of a primitive robotic fish. It either enhanced a behavior with pleasure consciousness, or repressed it with pain. That was the purpose of consciousness at this stage of evolution. But somewhere along the line consciousness formed an alliance with brain plasticity that allowed a new way of learning. The new learning would not require a biological mutation together with the long and painful demise of an old model. Instead consciousness, now allied with plasticity, directly represses undesirable neural pathways and enhances more desirable ones.

This, it appears, is the primary purpose of consciousness in the more advanced creatures in which it appears. Perhaps the above learning processes were present from the beginning. It is certainly possible that consciousness teemed up with brain plasticity when it first appeared in Robofish. That would not change the argument (Mould, 2009a) for each kind of experience (pain, pleasure, fear, vision, etc.) would still have to be introduced by mutation. Only then might the causally effective repression and enhancement properties of consciousness join with plasticity to permit learning in the modern sense.

\section{REFERENCES}

Hoshberg, L. R., et al. (2006). Neuronal ensemble control of prosthetic devices by a human with tetraplegia. Nature, 442, 164-71.

Kohler, I. (1951). Über Aufbau und Wandlungen der Wahmehmungswelt. Österreichische Akademie der Wissenschaften. Sitzungsberichte, philosophischhistorische Klasse, 227, 1-118.

Mould, R. A. (2009a). Evolution of Consciousness I. Activitas Nervosa Superior, 51, 83-86.

Mould, R. A. (2009b). Evolution of Consciousness II. Activitas Nervosa Superior, 51, 87-89

Noë, A. (2009). Out Of Our Heads, Why You Are Not Your Brain and Other Lessons from the Biology of Consciousness. New York: Hill and Wang.

O'Regan, J. K., \& Noë, A. (2001). A sensorimotor account of vision and visual consciousness. Behavioral and Brain Sciences, 24, 939-973, sect. 5.9 Evolved Consciousness 12. 